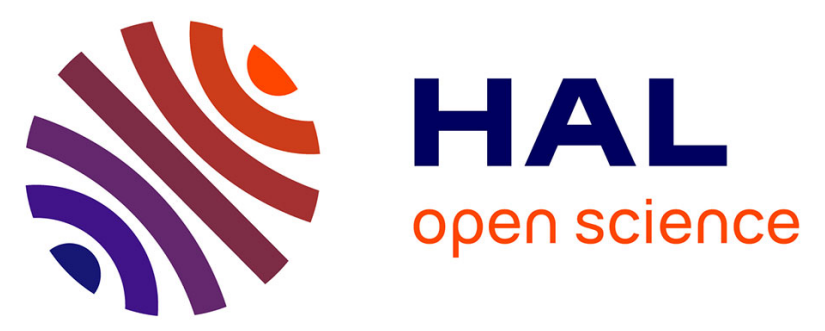

\title{
A millifluidic calorimeter with InfraRed thermography for the measurement of chemical reaction enthalpy and kinetics
}

Cindy Hany, Christophe Pradere, Jean Toutain, Jean-Christophe Batsale

\section{- To cite this version:}

Cindy Hany, Christophe Pradere, Jean Toutain, Jean-Christophe Batsale. A millifluidic calorimeter with InfraRed thermography for the measurement of chemical reaction enthalpy and kinetics. Quantitative InfraRed Thermography Journal, 2012, 5/2 (2), pp.211-229. 10.3166/qirt.5.211-229 . hal-00742252

\section{HAL Id: hal-00742252 \\ https://hal.science/hal-00742252}

Submitted on 16 Oct 2012

HAL is a multi-disciplinary open access archive for the deposit and dissemination of scientific research documents, whether they are published or not. The documents may come from teaching and research institutions in France or abroad, or from public or private research centers.
L'archive ouverte pluridisciplinaire HAL, est destinée au dépôt et à la diffusion de documents scientifiques de niveau recherche, publiés ou non, émanant des établissements d'enseignement et de recherche français ou étrangers, des laboratoires publics ou privés. 


\title{
A millifluidic calorimeter with InfraRed thermography for the measurement of chemical reaction enthalpy and kinetics
}

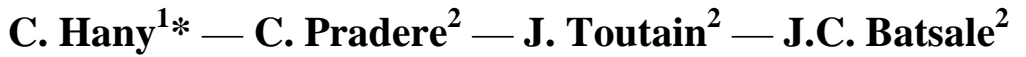 \\ ${ }^{1}$ Laboratoire du Futur (LOF), Université Bordeaux 1, UMR CNRS-Rhodia-UB1 \\ 5258, 33608 Pessac cedex, France \\ cindy.hany-exterieur@eu.rhodia.com \\ ${ }^{2}$ Laboratoire TRansfert Ecoulements FLuides et Energétique (TREFLE), Université \\ Bordeaux 1, UMR CNRS-ENSAM-UB1 8508, Esplanade des Arts et Métiers, 33405 \\ Talence, France \\ christophe.pradere@bordeaux.ensam.fr \\ toutain@enscpb.fr \\ jean-christophe.batsale@bordeaux.ensam.fr
}

\begin{abstract}
The aim of this work is to present an infrared calorimeter for the measurement of the kinetics and the enthalpy of high exothermic chemical reactions. The main idea is to use a millifluidic chip where the channel acts as a chemical reactor. An infrared camera is used to deduce the heat flux produced by the chemical reaction from the processing of temperature fields. Due to the size of the microchannel a small volume of reagents $(\mathrm{mL})$ is used. As the chemical reagents are injected by syringe pump, continuous experiments are performed with a very good control of the reagents mixing. A specific injection system enables to perform two flow configurations: co-flow and droplets. Thanks to the thermal isoperibolic conditions, the chemical reaction can be easily characterized with a previous specific calibration. Here, the kinetic and the enthalpy of a strong acid base reaction are monitored in co - flow configuration.

KEY WORDS: Calorimeter, Chemical Reaction, Enthalpy, Kinetics, Millifluidic, Temperature field processing.
\end{abstract}

QIRT Journal. Volume $\mathrm{X}-\mathrm{N}^{\circ} \mathrm{X} / 200 \mathrm{X}$, pages 1 to $\mathrm{n}$ 


\section{Introduction}

Microfluidic chemical reactors are one of the very promising tools given by MEMS (Micro-Electro-Mechanical System). One application of MEMS consists in investigating the chemical reaction in continuous flow in microchannel. These systems are used to study kinetics of chemical reaction by on-line spectroscopy methods. Some studies consist in using Raman spectroscopy to probe the concentration profile in the microchannel (Cristobal et al., 2006; Sarrazin et al., 2008). Another one measures the kinetics of a chemical reaction with a UV - visible spectrophotometer (Engl et al., 2008). These techniques provide quite accurate results. In order to decrease the experiment time, we investigate the use of calorimetry adapted to such microsystems.

The knowledge of kinetics and thermodynamics of chemical reactions is required for chemical process development and safety. We know that both phenomena, i.e., heat transfer and chemical kinetics, occur in chemical reactions. One way to control and measure those phenomena is calorimetry. The characterisation of exothermic chemical reaction is well developed and different kinds of calorimeter already exist (Zogg et al., 2004). Nevertheless, they are not simultaneously adapted to the use of microvolumes, high thermal sensitivity, good control of the reagent mixing and quasi isothermal conditions.

To this end, the recent development of MEMS (Micro-Electro-Mechanical Systems) allows the investigation of new approaches to measure the enthalpy of chemical reactions. The main advantage is the small size of the microchannels which permits the mixing control of the reactants. Due to the microchannel size, the flow is laminar with low Reynolds number $(<100)$. It is also convenient to use a small amount of reagents to be able to easily and quickly test a large number of reaction configuration. The measurement of the temperature at a small scale represents the main difficulty. However the temperature information in such devices is among the most important parameter. Today, only a few studies deal with this. One method consists in developing localised temperature microsensors (Köhler et al., 1998). A second one measures the global heat flux produced by a chemical reaction in a microfluidic chip (Hany et al., 2008). Another one consists in measuring the infrared emission field of a surface without contact and leads to a qualitative analysis (Mollmann et al., 2004). Due to the poor knowledge of the radiative properties (spectral emissivity, transparency, etc.) of the investigated surface, the accuracy of the absolute temperature field is difficult. A recent study shows that the quantitative interpretation of such fields is possible with previous and simplified heat transfer model (Pradere et al., 2004). As the classical microfluidic chips are made of PDMS: PolyDiMethylSiloxane (thermal insulator), with a substrate of glass or silicon (thermal conductor). The thermal diffusion is very important in the substrate and cannot be neglected.

This work is devoted to the development of a new flow calorimeter for a non contact temperature field measurement by using an infrared camera. A micro millifluidic chip is designed by a PFA (Perfluoroalkoxy) tubing and an aluminium plate. Contrary to the classical chips (Pradere et al., 2004), the use of PFA tubing (insulator) and aluminium plate (conductor) allows to be isoperibolic and to simplify 
the heat transfer. The purpose is to obtain enthalpy and kinetics to characterize chemical reaction. Firstly, the experimental device principle is described. The isoperibolic chip and the injection system of the reagents runnings are explained. Secondly, a thermal calibration is presented. The camera is calibrated by Joule effect in order to define the conversion coefficient. The chip is also calibrated to estimate the heat losses of the system. Finally, the method of estimation of the reaction enthalpy is developed and the enthalpy and the kinetics of a well-known strong acid base reaction are determined in co-flow configuration.

\section{Experimental device}

\subsection{Isoperibolic chip}

A millifluidic chip, represented in figure 1, is used to perform the chemical reaction. The chip consists in PFA (Perfluoroalkoxy) tubing and an aluminium plate. As the PFA is a good thermal insulator $\left(\lambda=0.25 \mathrm{~W} \cdot \mathrm{M}^{-1} \cdot \mathrm{K}^{-1}\right)$ and the aluminium plate a good thermal conductor $\left(\lambda=237 \mathrm{~W} \cdot \mathrm{M}^{-1} \cdot \mathrm{K}^{-1}\right)$, the tubing is assumed as isoperibolic. In fact, the temperature of the aluminium plate $\left(T_{c}\right)$ is kept constant. Thus the radial heat transfer between the reaction area and the aluminium plate is controlled by the wall of the tubing (PFA insulator). This wall acts as a simple thermal resistance.

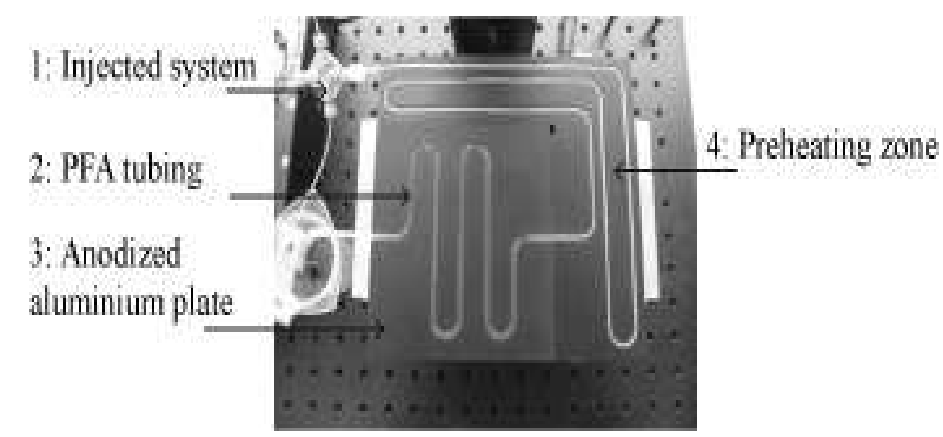

Figure 1. Picture of the isoperibolic chip

The aluminium plate of $12 \mathrm{~cm} \mathrm{X} 12 \mathrm{~cm} \mathrm{X} 8 \mathrm{~mm}$ includes a serpentine groove to place the reaction tubing. This plate allows to control the temperature around the channel. A heating or cooling Peltier system allows setting different temperatures $\left(T_{c}\right)$. A surface treatment is realized to obtain a black body.

In order to control the reagents mixing starting point, very little tubing are introduced in the PFA ones thanks to a cross junction. Each tubing contains only one chemical reagent which can be preheated (figure 1,4 ), if necessary. 
This injection system, represented in figure 2, allows to perform chemical reaction in co-flow (a) or droplets (b). The mixing area starting point is controlled by adjusting the position of the reagents tubing. The typical dimensions of the PFA tubing are $3.17 \mathrm{~mm}$ for the outer diameter and 0.2 to $2.6 \mathrm{~mm}$ for the inner one.

During the following experiments, the inner diameter of the PFA tubing is equal to $1.6 \mathrm{~mm}$. For the reagents tubing, the outer diameter is about $800 \mu \mathrm{m}$ and the inner one is equal to $500 \mu \mathrm{m}$.
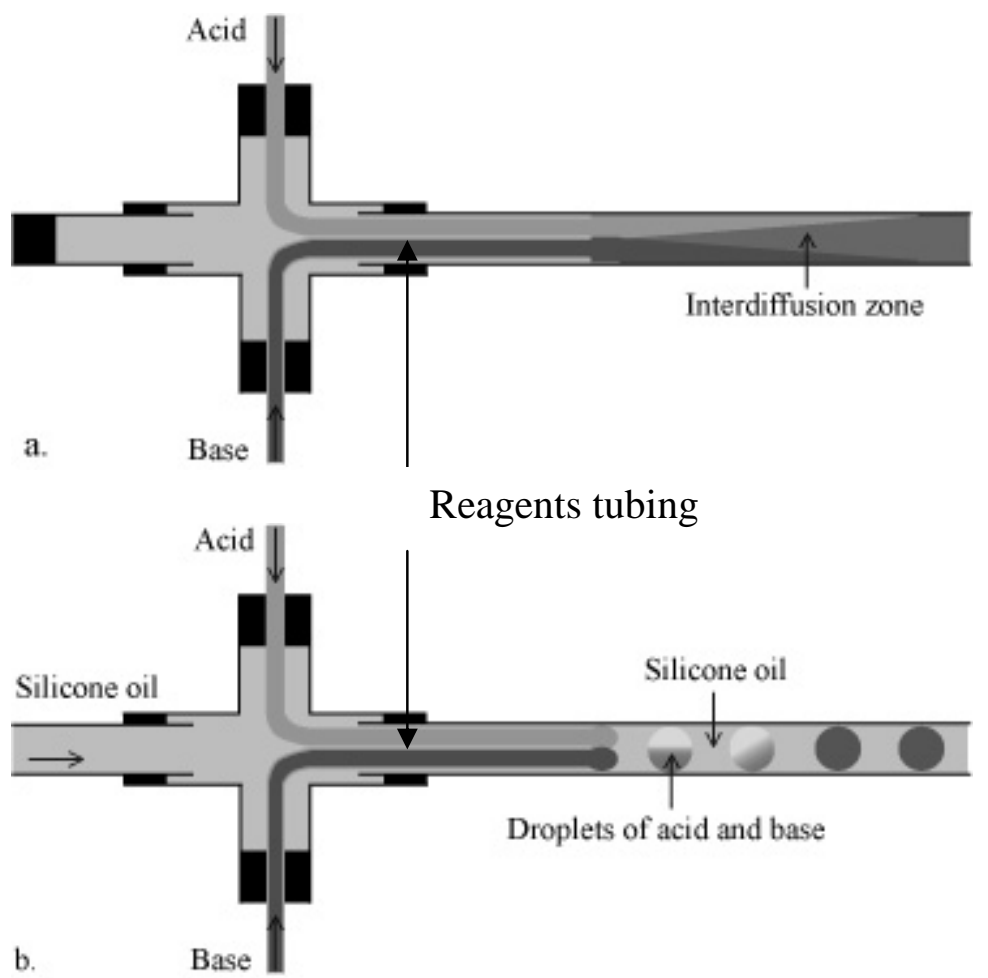

Figure 2. Scheme of the reactants injection in co-flow (a) and droplets (b) configurations

In such device the Reynolds number is low $(<100)$, so the flow is laminar. In the case of co-flow configuration, the reagent mixing occurs by species diffusion and the thermal heat transfer occurs in steady state. The interdiffusion area was measured and studied by several investigations (Kamholz et al., 1999; Ismagilov et al., 2001; Salmon et al., 2005). In droplet configuration, the reagent mixing is much faster than the co-flow configuration and the thermal behaviour is in transient state. This is due to the velocity and the recirculations inside the droplet (Sarrazin et al.; 2006). 


\subsection{Measurements device}

The measurement device is shown in figure 3. An infrared camera CEDIP, JADE MWIR J550, In-Sb focal plane array of detectors $(1.5-5.2 \mu \mathrm{m}, 240 * 320$ pixels, pitch $30 \mu \mathrm{m}$ ) with a $25 \mathrm{~mm}$ objective MWIR F/2 (space resolution about $200 \mu \mathrm{m}$ ) is placed in front of the isoperibolic chip. The chemical reaction occurs in the PFA tubing and temperature field is measured by this IR camera.
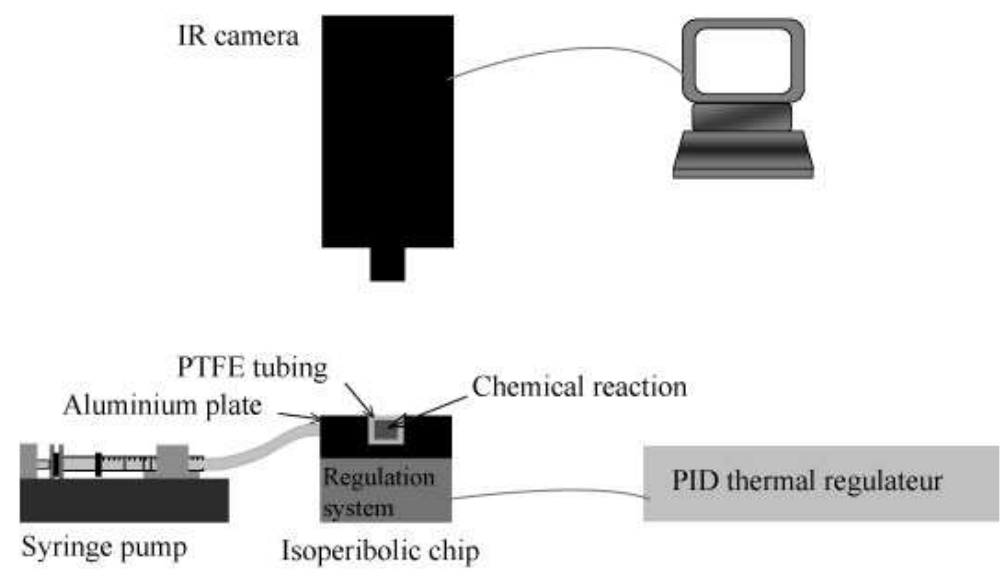

Figure 3. Scheme of the set up

The temperature regulation system is an Agilent device using a PID regulation. The temperature is imposed by using Peltier elements. With this apparatus the setting temperature can vary from 5 to $90{ }^{\circ} \mathrm{C}$ with a sensitivity of $0.1{ }^{\circ} \mathrm{C}$.

The measurement principle consists to inject the reagents in the millifluidic chip by syringe pump (Cetoni, Nemesys) at various flow rates from 2 to $100 \mathrm{~mL} \cdot \mathrm{h}^{-1}$. Thus the chemical reaction occurs and is observed by the IR camera at thermal steady state. The PFA is black painted in order to be opaque to the infrared wavelength. The aluminium plate is also anodized to avoid light reflection on the surface.

Even with such precaution, we assumed that the measured temperatures fields are only proportional to absolute temperatures in the PFA tubing. Then, in order to avoid non uniform effects (emissivity defects or reflection disturbance) which are harmful for quantitative measurements we proposed a previous camera calibration.

\section{Calibration of the device}




\subsection{Camera calibration}

The camera is calibrated in order to link the heat flux dissipated inside the PFA tubing and the temperature measured at its surface. For that purpose, an electrical conductive tin wire is introduced inside the chip channel (figure 4). Then, the channel is full filled by water and various electrical powers, from 0.25 to $3 \mathrm{~W}$, are applied to the tin wire. When the steady state is reached, the temperature variation between the channel $\left(T_{E}\right)$ and the aluminium plate $\left(T_{c}\right)$ is measured thanks to the IR camera.

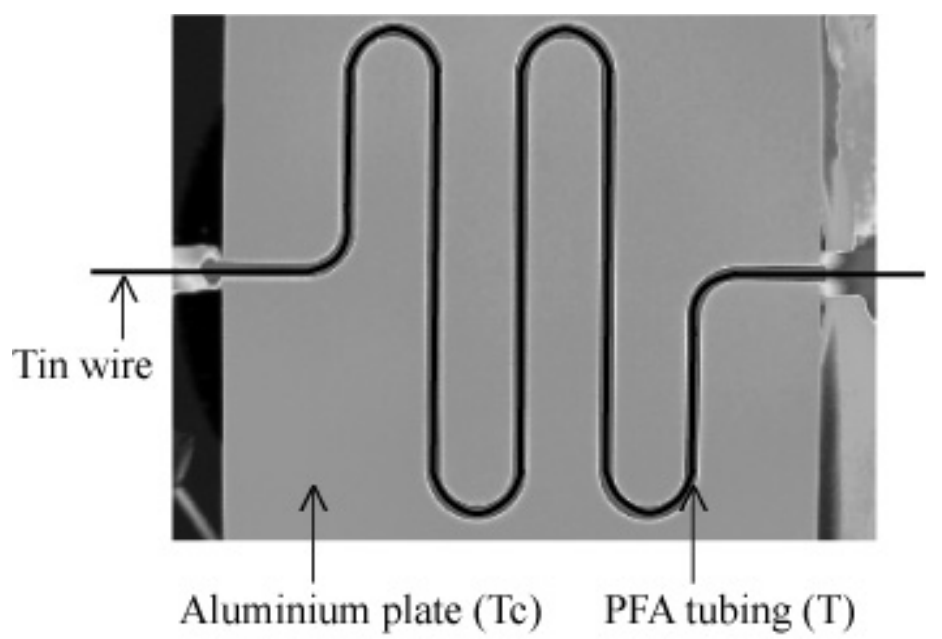

Figure 4. IR image of the chip during the electrical Joule effect calibration of the camera

In this disposition, the relation between injected power and the non-calibrated temperature rises can be determined according to the following energy balance:

$$
P=h \pi d L\left(T_{E}-T_{c}\right)
$$

Where, $P$ is the power dissipated by Joule effect $(\mathrm{W}), d$ is the tubing diameter $(\mathrm{m}), L$ is the tubing length $(\mathrm{m}), h$ is the global heat transfer coefficient $\left(\mathrm{W} \cdot \mathrm{DL}^{-1} \cdot \mathrm{m}^{-2}\right), T_{E}$ is the tubing temperature (DL: Digital Level) and $T_{c}$ is the aluminium plate temperature (DL).

Figure 5 represents the injected power versus the temperature variation between the PFA tubing and the aluminium plate. The results are linear. Thus the calibration coefficient $H_{\mathrm{c}}$ between the heat flux and the temperature in Digital Level is determined by the slope: $H_{c}=h \pi d L=1.38 \mathrm{~mW} \cdot \mathrm{DL}^{-1}$ at room temperature. As the 
length $L=47 \mathrm{~cm}$ and the PFA inner tubing diameter $d=1.6 \mathrm{~mm}$ are well know, we can deduced the heat transfer coefficient $h=480 \mathrm{~mW} \cdot \mathrm{DL}^{-1} \cdot \mathrm{m}^{-2}$.

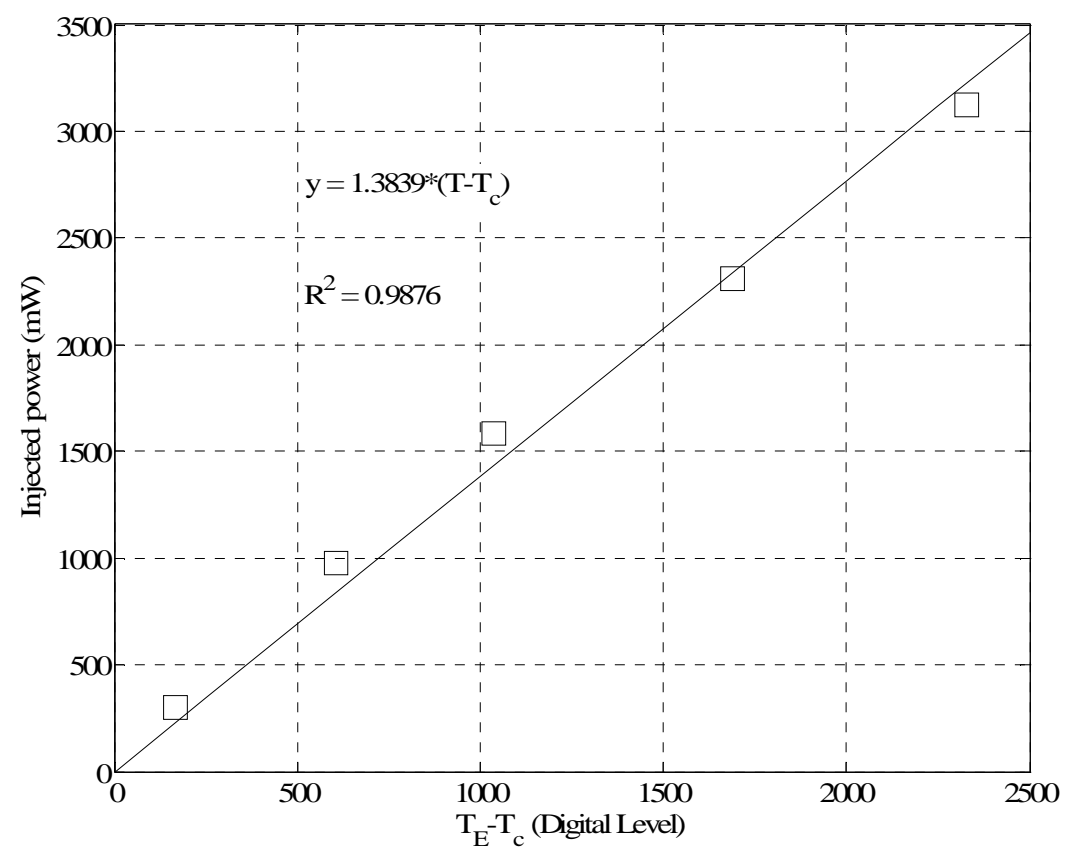

Figure 5. Injected power versus the temperature variation between the PFA tubing $\left(T_{E}\right)$ and the aluminium plate $\left(T_{C}\right)$

\subsection{Chip calibration}

The chip calibration is performed in order to identify the heat losses (or thermal resistance), between the fluid under flow inside the channel, and the surrounding (aluminium plate and ambient air). The chip calibration is achieved by maintaining a constant temperature (here $T_{c}=10^{\circ} \mathrm{C}$ ) thanks to a regulation system. The fluids (without chemical reaction) are injected by the syringe pump at different flow rates from 2 to $60 \mathrm{~mL} \cdot \mathrm{h}^{-1}$. 


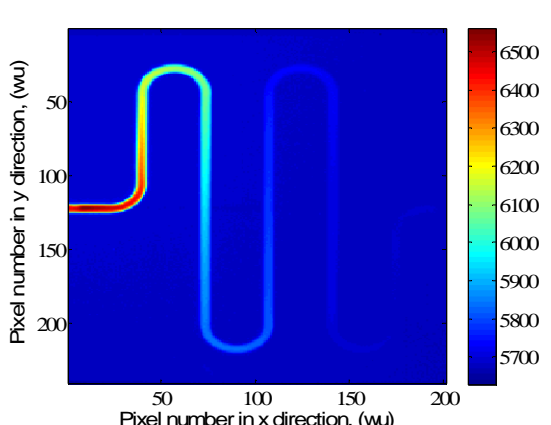

(a)

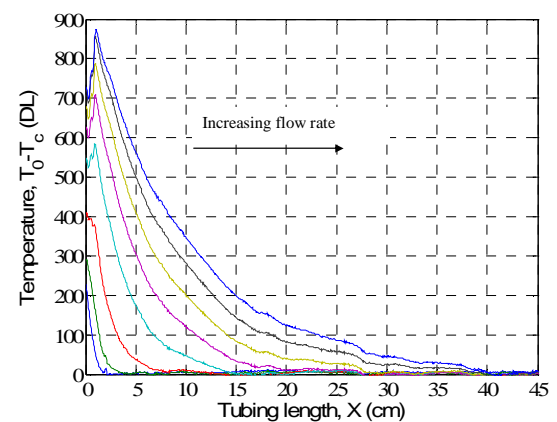

(b)

Figure 6. a) IR image of the fluid without chemical reaction at $Q=120 \mathrm{~mL} / \mathrm{h}$ and $\left.T_{c}=10^{\circ} \mathrm{C}, b\right)$ Tubing temperature $\left(T_{0}-T_{c}\right)$ versus tubing length for various flow rates:

When the steady state is reached, an IR image is taken (figure 6a). Then, the tubing temperature $\left(T_{0}\right)$ is plotted versus the tubing length at various flow rates by the use of a specific algorithm developed under Matlab ${ }^{\mathrm{TM}}$ environment (figure $6 \mathrm{~b}$ ). This algorithm applied image processing in order to extract the temperature fields of the channel in curvilinear coordinates.

As we are in continuous flow and in an isoperibolic chip without chemical reaction, the temperature profile of the tubing is assumed to be described by the following simplified 1D energy balance.

$\frac{d T_{0}}{d x}=-H\left(T_{0}-T_{c}\right)$

with: $H=\frac{h \pi d}{\rho C_{p} Q}$

and where: $h$ is the heat losses coefficient measured in the electrical calibration (W.DL $\left.{ }^{-1} \cdot \mathrm{m}^{-2}\right), \rho$ is the mass density $\left(\mathrm{kg} \cdot \mathrm{m}^{-3}\right), C_{p}$ is the specific heat $\left(\mathrm{J}^{\mathrm{kg}} \mathrm{kg}^{-1} \cdot \mathrm{K}^{-1}\right), Q$ is the imposed flow rate $\left(\mathrm{m}^{3} \cdot \mathrm{s}^{-1}\right), d$ is the well known inner tubing diameter $(\mathrm{m}), x$ is the distance along the tubing $(\mathrm{m})$ and $T_{0}$ is the tubing temperature without chemical reaction $(\mathrm{DL})$.

The analytical solution of equation [2] is established by:

$\frac{T_{o}(x)-T_{c}}{T_{c}}=-\exp (-H x)$

and the heat losses characteristic length $(H)$ is estimated by a linear regression on the semi log plot (figure 7) of the expression [3]. 


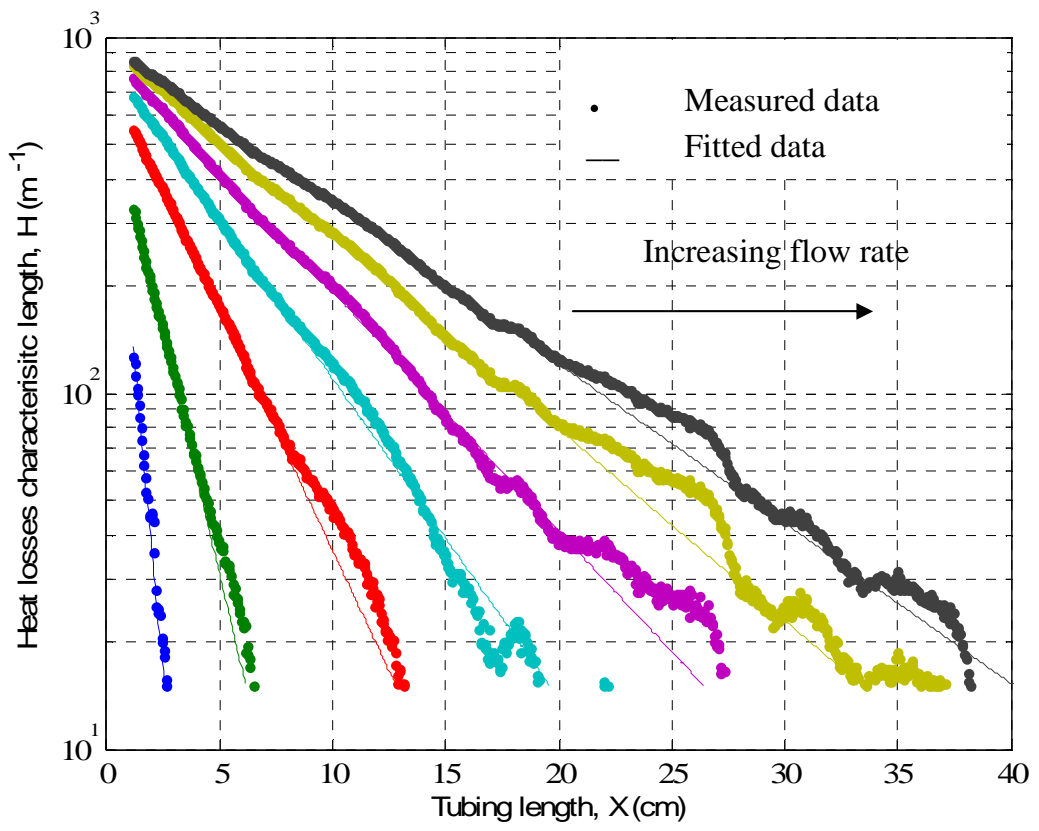

Figure 7. Estimation of Heat losses characteristic length at $T_{c}=10^{\circ} \mathrm{C}$ for various flow rates: $2,4,10,20,30,40,50,60 \mathrm{~mL} \cdot \mathrm{h}^{-1}$

From the results obtained figure 7 and the relation [3], we can verify that evaluated $H$ is proportional to the fluid flow rate at the power -1 . For that, the experimental plot $\log (H)=\mathrm{f}(\log (Q)$ is represented in figure 8 . Indeed, the experimental data, obtained by the previous experiments, are perfectly aligned. The measured slope is equal to -0.989 that is very close to -1 . 


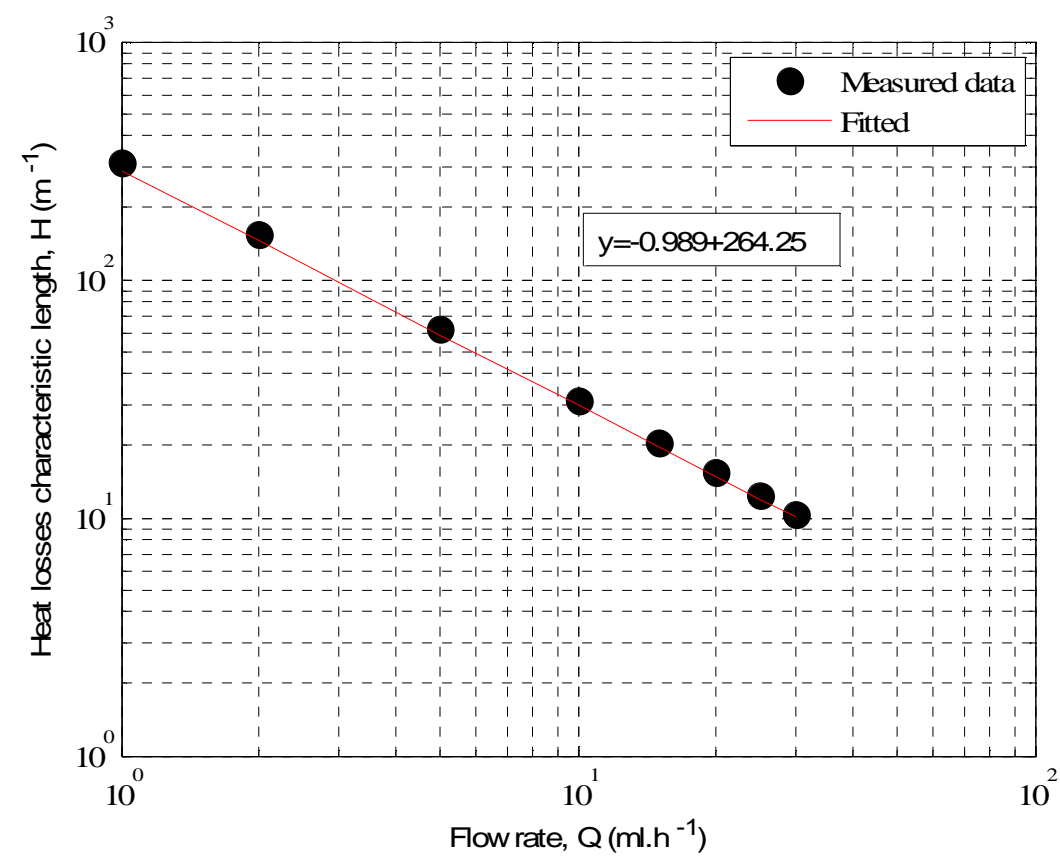

Figure 8. Validation of the isoperibolic chip calibration, $\log (H)=f(\log (Q))$

\section{Measurement on chemical reaction}

\subsection{Method for the estimation of the kinetic and the enthalpy}

When a chemical reaction under continuous flow occurs in the tubing, the energy balance is given by:

$\rho C_{p} Q \frac{d T(x)}{d x}=\phi(x)-h \pi d\left(T(x)-T_{c}\right)$

where, $T$ is the tubing temperature with the chemical reaction (DL) and $\phi$ is the heat flux per unit length $\left(\mathrm{W} \cdot \mathrm{m}^{-1}\right)$.

As the injected chemical products are not necessary at the temperature $\left(T_{C}\right)$ of the aluminium plate when the chemical reaction begins, the superposition principle is applied to separate the fluid contribution from the chemical one. For that, we assume that the thermal properties of the fluids are constants because the temperature variations are always lower than $5^{\circ} \mathrm{C}$. The following definition $T_{c h}=T-T_{0}$ and the use 
of the two-energy balance equations written before, one related to the flow without chemical reaction [2] and the other one related to the flow with chemical reaction [4], allow to establish that $T_{c h}$ is related to the heat flux by:

$$
\rho C_{p} Q \frac{d T_{c h}(x)}{d x}=\phi(x)-h \pi d\left(T_{c h}(x)-T_{c}\right)
$$

where, $T_{c h}(\mathrm{DL})$ corresponds to the temperature due to the chemical reaction only. From the knowledge of the calibration coefficient, the heat losses characteristic length and the equation [5], the kinetic of the chemical reaction (i.e. $\phi(x)$ ) is estimated:

$$
\phi(x)=\frac{H_{c}}{L}\left[\left(T_{c h}(x)-T_{c}\right)+\frac{d T_{c h}(x)}{d x} \frac{1}{H}\right]
$$

where: $T_{c h}$ and $T_{c}$ are the measured temperatures, $H_{c}$ and $H$ are the calibration coefficient and the heat losses characteristic length estimated before. For this estimation, the spatial derivative is calculated with a numerical finite difference schema.

Finally, the total heat flux $\Phi$ (i.e. the reaction enthalpy) dissipated by the chemical reaction is characterized by integration of the local heat flux [6] along the tubing length:

$$
\Phi=\int_{x=0}^{L} \phi(x) d x
$$

\subsection{Application to an acid - base reaction}

The kinetic and enthalpy estimation in co-flow configuration of a well-known high exothermic reaction between a strong acid (HCL) and a strong base $(\mathrm{NaOH})$ is performed in order to validate this device. The concentration of the two reagents is the same and equal to $0.2 \mathrm{~mol} . \mathrm{L}^{-1}$. The experiments are realized at various flow rates from 2 to $60 \mathrm{~mL} \cdot \mathrm{h}^{-1}$ for each products. For all experiments, the stoechiometric proportions are applied. The setting temperature is the same as during the chip calibration $\left(T_{c}=10^{\circ} \mathrm{C}\right)$. When the steady state is reached, an IR image is taken (figure 9.a). 


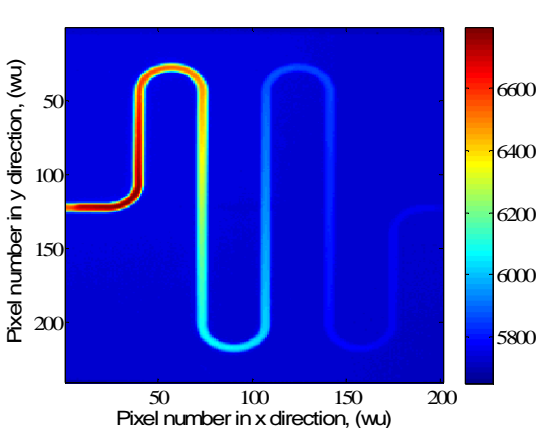

(a)

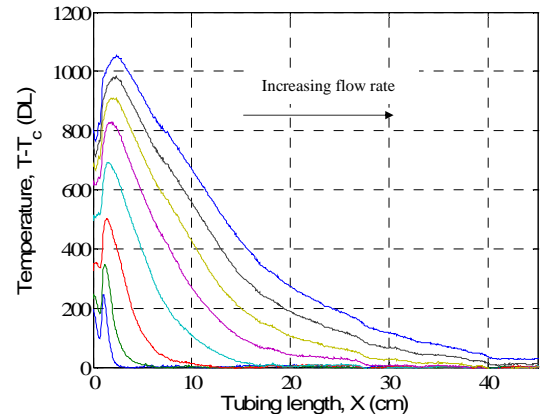

(b)

Figure 9. a) IR image of the fluid with chemical reaction (corresponding to T) at $Q_{a}=Q_{b}=60 \mathrm{~mL} \cdot \mathrm{h}^{-1}$ and $\left.T_{c}=10^{\circ} \mathrm{C}, \mathrm{b}\right)$ temperature $\left(T-T_{c}\right)$ versus tubing length for various flow rates: $2,4,10,20,30,40,50,60 \mathrm{~mL} \cdot \mathrm{h}^{-1}$

In order to obtain the temperature profiles from the reaction only, the superposition principle [5] is processed. The IR image corresponding to the fluid flow only (figure 6) is substracted to the IR image corresponding to the fluid flow where the chemical reaction occurs for each flow rate (figure 9). Thus the figure 10.a is obtained which correspond to the temperature profiles shown in figure 10.b of the chemical reaction only.

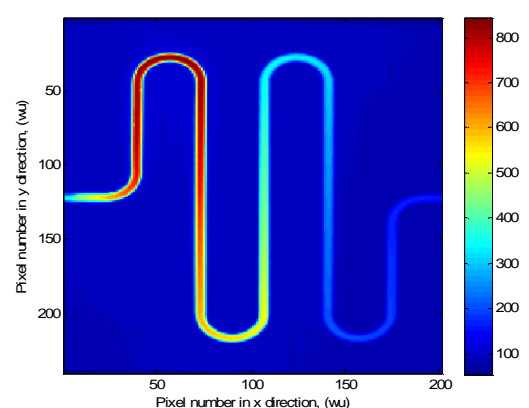

(a)

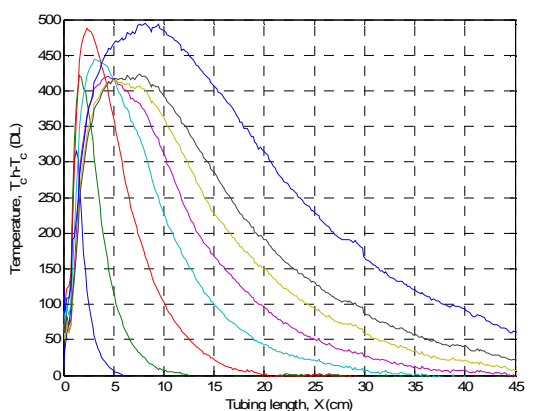

(b)

Figure 10. a) IR image of chip corresponding to $T_{c h}=T-T_{0}$ at $Q_{a}=Q_{b}=60 \mathrm{~mL} \cdot \mathrm{h}^{-1}$ and $\left.T_{c}=10^{\circ} \mathrm{C} ; \mathrm{b}\right)$, temperature due to the chemical reaction $\left(T_{c h}-T_{c}\right)$ versus tubing length for various flow rates: 2, 4, 10, 20, 30, 40, 50, $60 \mathrm{~mL} . \mathrm{h}^{-1}$

As the calibration coefficient $\left(H_{c}\right)$ and the heat losses characteristic length $(H)$ are known, the integration of the dissipated heat flux (corresponding to the chemical 
reaction) can be estimated thanks to the equation [6]. Due to the steady state of the millifluidic configuration, a direct relation between space and time is given by $x=Q t / S$, where $S$ is the inner tubing section. For this reason, the local estimated heat flux can also be expressed as function of time and defined as follow:

$\phi(t)=C Q \Delta H \frac{d \alpha}{d t}$

Where $C$ is the concentration of the limited reagents $\left(\right.$ mol. $\left.\mathrm{L}^{-1}\right), \Delta H$ is the reaction enthalpy $\left(\mathrm{J} . \mathrm{mol}^{-1}\right), d \alpha / d t$ is the conversion rate $\left(\mathrm{s}^{-1}\right)$ and $t$ is the time $(\mathrm{s})$.

Figure 11 shows the heat source versus time (i.e. the kinetic of the reaction) for the different flow rates. As shown in expression [8], the conversion rate for this reaction is independent of the molar flow rate. That is why for the different flow rate, the temporal evolution of the heat flux is similar. Otherwise (figure 11), when the flow rate increases, the level of the heat flux also increases. This is due to the elevation of the number of mole injected in the tubing.

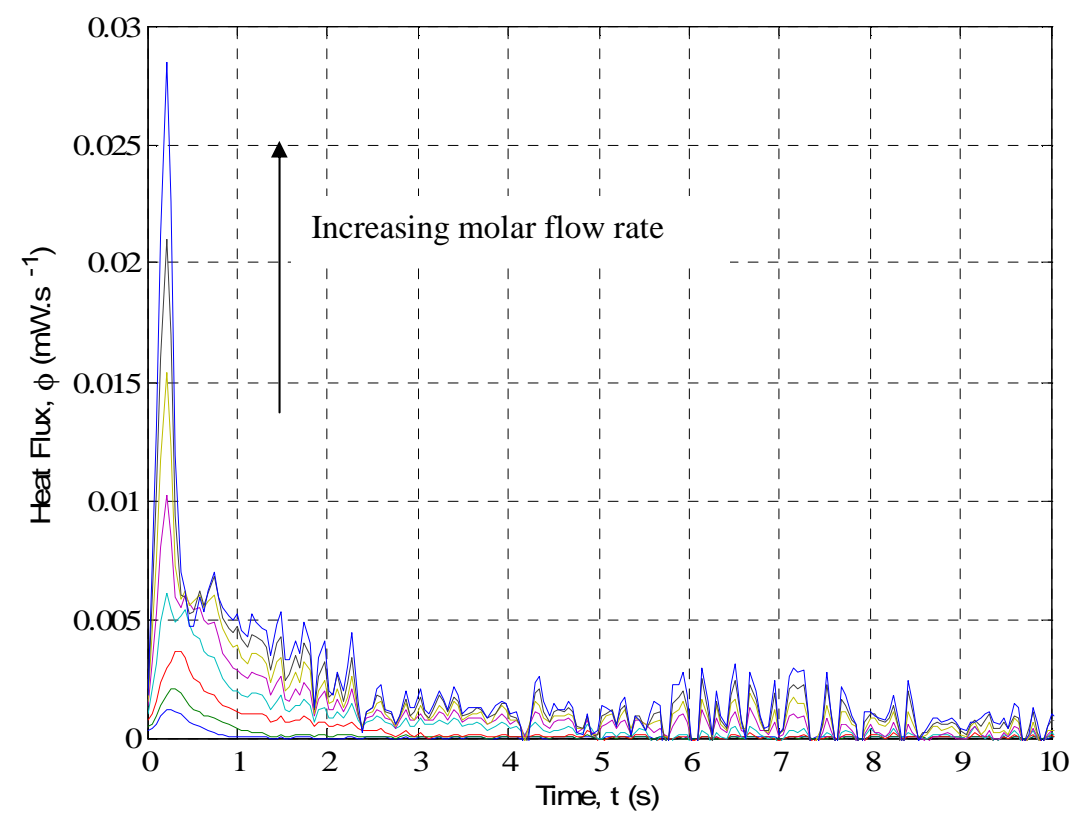

Figure 11. Heat source from the chemical reaction as function of time for various flow rates: 2, 4, 10, 20, 30, 40, 50, $60 \mathrm{~mL} \cdot \mathrm{h}^{-1}$ 
According to the equations [7] and [8] and assuming that the reaction is complete (very high reaction rate), the total heat flux of each flow rate can be determined by integration of all heat sources of the figure 11. As the measurements are performed at one initial concentration and for different flow rates, the relation [9] could be written:

$\Phi=Q_{m} \Delta H$

$Q_{m}=C Q$

To determine the enthalpy of the chemical reaction, the total exothermic flux $(\Phi)$ is plotted versus the molar flow rate $\left(Q_{m}\right)$ in the figure 12 . The results are accurately aligned and the slope, which corresponds directly to the reaction enthalpy, is equal to $57.6 \mathrm{~kJ} \mathrm{~mol}^{-1}$. This result is in a good agreement with the literature value of $56 \mathrm{~kJ}^{\mathrm{mol}}{ }^{-1}$ (Lide, 1991-1992). This allows the validation of the flow calorimeter.

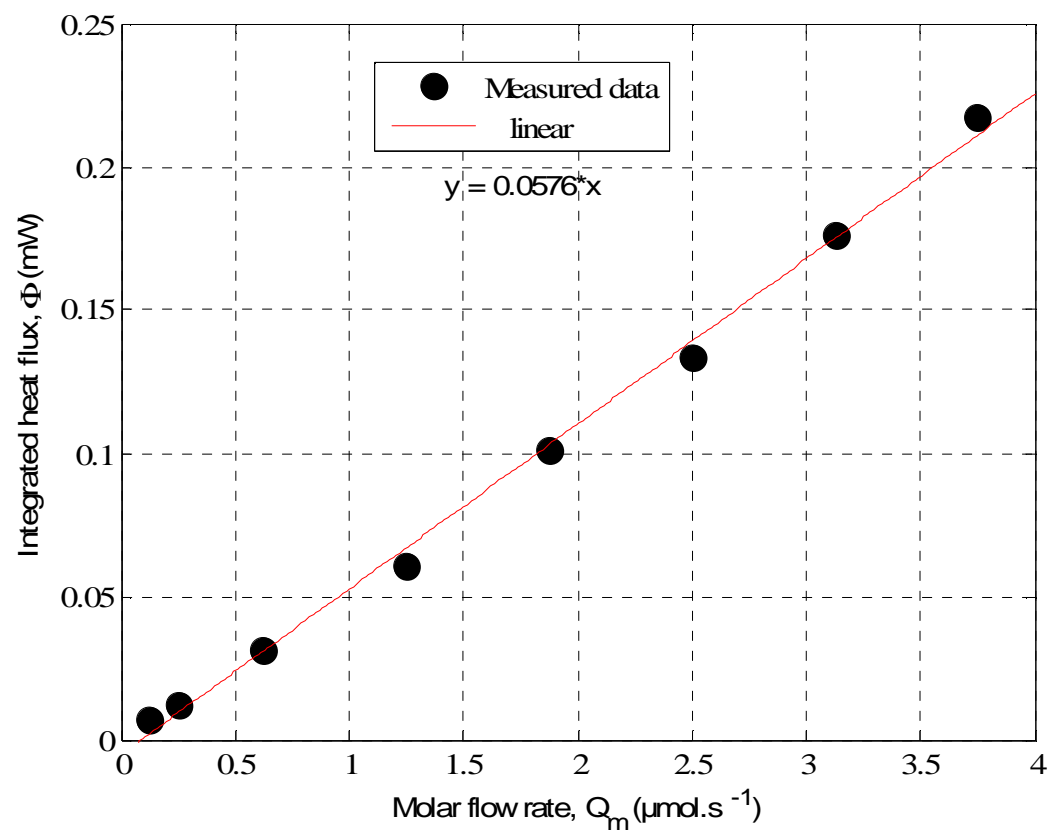

Figure 12. Integrated heat flux versus molar flow rate

\section{Conclusion and outlooks}


To conclude, it is relevant to note that we developed and validated a millifluidic infrared calorimeter under continuous flow for the measurement of the enthalpy and kinetics of chemical reaction.

In this paper, an isoperibolic chip has been designed and allows to simplify and calibrate the lateral heat losses in comparison with the classical microfluidic chips. This device composed of the isoperibolic chip and an infrared camera has been validated by the characterisation of a strong acid base reaction.

The advantages of this system are summarized:

- the manufacturing of the chips makes possible to obtain isoperibolic conditions with various geometries of reactor (sizes and volumes).

- the infrared camera offers a good sensitivity and a direct measurement of the heat flux. A local analyse is also possible to study kinetics.

- thanks to the injection system, a large range of residence times and concentrations can be swept.

- the global required time to investigate the reaction enthalpy, versus a large range of concentration (10 flow rates), is around 1 hour which is very short.

This work should be extended in several ways. First, a chemical reaction will be studied in droplet configuration. The droplet flow is very useful in case of very high exothermic reaction. In fact, the small volume and the important exchange surface of the droplets give very low temperature increases. These advantages are interesting for safety studies. Second, the characterisation of other chemical reactions such as polymerisation will be investigated. Third, the concentration measurement by the use of Raman spectroscopy, interferometer or multi spectral method will be coupled with our calorimeter.

\section{References}

Cristobal G., Arbouet L., Sarrazin F., Talaga D., Bruneel J-L., Joanicot M. and Servant L., "On-line laser Raman spectroscopic probing of droplets engineered in microfluidic devices", Lab Chip, 6,1140-1146, 2006.

Engl W., Tachibana M., Colin A. and Panizza P., "A droplet-based high-throughput tubular platform to extract rate constants of slow chemical reactions", Chemical Engineering Science, 63 (6): 1692-1695, 2008.

Hany C., Pradere C., Toutain J., Batsale J.C. and Joanicot M., "A microfluidic calorimeter for the measurement of the enthalpy and the kinetics of chemical reactions from 5 to $90^{\circ} \mathrm{C}$ ", in proceedings of AICHE spring meeting IMRET, New Orleans, 2008. 
Ismagilov R.F., Rosmarin D., Kenis P.J.A., Chiu D.T., Zhang W., Stone H.A. and Whitesides G.M., "Pressure-driven laminar flow in tangential microchannels: an elastomeric microfluidic switch", Analytical Chemistry, 73, 4682-4687, 2001.

Kamholz A.E., Weigl B.H., Finlayson B.A. and Yager P., "Quantitative analysis of molecular interaction in a microfluidic channel: the T-sensor", Analytical Chemistry, 71, 5340-5347, 1999.

Köhler J.M. and Zieren M., "Chip reactor for microfluidic calorimetry", Thermochimica Acta, 310, 25-35, 1998.

Lide D.R.; Handbook of Chemistry and Physics, CRC Press, $72^{\mathrm{ND}}$ Edition, p. 5-1, 1991-1992.

Mollmann K. P., Lutz N. and Vollmer M., "Thermography of Microsystems", Inframation proceedings, ITC 104A, 07-27, 2004.

Pradere C., Joanicot M., Batsale J.C., Toutain J. and Gourdon C., "Processing of temperature field in chemical microreactors with infrared thermography", QIRT Journal Volume 3, ${ }^{\circ} 1$, pages 117 to 135, 2004.

Salmon J.B., Dubrocq C., Tabeling P., Charier S., Alcor D., Jullien L. and Ferrage F., "An approach to extract rate constants from reaction - diffusion dynamics in a microchannel”, Analytical Chemistry, 77, 3417-3224, 2005.

Sarrazin F., Loubière K., Prat L., Gourdon C., Bonometti T. and Magnaudet J., "Experimental and numerical study of droplets hydrodynamics in microchannels", AICHE Journal, Vol. 52, No.12, 2006.

Sarrazin F., Salmon J-B., Talaga D. and Servant L., "Chemical reaction imaging within microfluidic devices using confocal Raman spectroscopy: the case of water and deuterium oxide as the model system", Analytical chemistry, Vol. 80,No. 5,March 1, 2008.

Zogg A., Stoessel F., Fischer U. and Hungerbühler K., "Isothermal reaction calorimetry as a tool for kinetic analysis", Thermochimica Acta, 419, 1-17, 2004. 